\title{
LEARNING DISABILITIES IN NEUROFIBROMATOSIS TYPE 1
}

The frequency of specific leaning disabilities (SLD) in neurofibromatosis type 1 (NF1) was determined in a cohort of 81 patients (43 males, 38 females; mean age 11 years 6 months; age range 8-16) followed at Children's Hospital, Westmead, NSW, Australia. Academic underachievement occurred in $42(52 \%)$ of NF1 children, 3.5 -fold more frequently than in a control group. One or more SLDs was present in $16(20 \%) ; 7$ had reading disability, 7 spelling, and 8 a math disability. Of the $52 \%$ with academic difficulties, one third had a general learning disability (GLD) associated with IQ deficit. Only males with NF1 were at significant risk for SLD; Verbal IQ<Performance IQ discrepancy was predictive of SLD. NF1 patients with no SLD or GLD had normal IQ scores and average academic achievement but minor difficulties in sustained attention and visuospatial skills. ADHD was present in 31 (38\%) NF1 children in the total group compared to $12 \%$ of 49 controls without NF1. The increase in frequency of ADHD was similar in those with comorbid SLD or GLD and lower but still significant in NF1 patients with no LD. (Hyman SL, Shores EA, North KN. Learning disabilities in children with neurofibromatosis type 1: subtypes, cognitive profile, and attention-deficit-hyperactivity disorder. Dev Med Child Neurol Dec 2006;48:973-977). (Respond: Kathryn N North MD, Clinical School, The Children's Hospital, Locked Bag 4001, Westmead, NSW 2145, Australia).

COMMENT. Children with NF1 have a $52 \%$ incidence of academic underachievement, and $20 \%$ have a SLD. One third of patients with LD have a GLD associated with a lowered IQ. Comorbid SLD and NF1 occur almost always in males, and in the above study, their verbal IQ was lower than performance IQ. The authors are examining VIQ $<$ PIQ discrepancies in male preschool children as early predictors of risk of SLD. These findings are different from those of Eliason MJ (Neurofibromatosis 1988;1:17-25) who reported a preponderance of nonverbal learning problems (visual perceptual deficits) in children with NF1.

\section{SEIZURE DISORDERS}

\section{DIAGNOSTIC ASSESSMENT OF STATUS EPILEPTICUS}

Diagnostic methods of assessment of the child with status epilepticus (SE) are outlined following an evidence-based review of literature by the Quality Standards Subcommittee of the AAN and Practice Committee of the CNS. SE is a seizure of at least 30 min duration or 2 or more sequential seizures without full recovery of consciousness between seizures lasting $30 \mathrm{~min}$. SE is classified by seizure type (focal, generalized or indeterminate) and etiology: 1) acute symptomatic, during an acute illness (26\%); 2) remote symptomatic, a prior CNS insult with chronic encephalopathy or malformation and no acute illness (33\%); 3) progressive encephalopathy (3\%); 4) febrile illness, with no direct CNS infection (22\%); and 5) cryptogenic, with no definable cause ( $15 \%)$. The etiologic incidence figures were obtained from 20 class III studies of 2,093 children. Laboratory studies were abnormal in $2.5 \%$, CNS infection in $12.8 \%$, AED levels were low in $32 \%$. An inborn error of metabolism was 